PROCEEDINGS OF THE

AMERICAN MATHEMATICAL SOCIETY

Volume 135, Number 10, October 2007, Pages 3171-3179

S 0002-9939(07)08897-1

Article electronically published on May 10, 2007

\title{
TRANSPORT \\ IN THE ONE-DIMENSIONAL SCHRÖDINGER EQUATION
}

\author{
MICHAEL GOLDBERG
}

(Communicated by David S. Tartakoff)

\begin{abstract}
We prove a dispersive estimate for the Schrödinger equation on the real line, mapping between weighted $L^{p}$ spaces with stronger time-decay $\left(|t|^{-\frac{3}{2}}\right.$ versus $\left.|t|^{-\frac{1}{2}}\right)$ than is possible on unweighted spaces. To satisfy this bound, the long-term behavior of solutions must include transport away from the origin. Our primary requirements are that $\langle x\rangle^{3} V$ be integrable and $-\Delta+V$ not have a resonance at zero energy. If a resonance is present (for example, in the free case), similar estimates are valid after projecting away from a rank-one subspace corresponding to the resonance.
\end{abstract}

In one dimension, the linear propagator of the free Schrödinger equation is given by the explicit convolution

$$
e^{-i t \Delta} \psi(x)=\frac{1}{\sqrt{-4 \pi i t}} \int_{\mathbb{R}} e^{-i \frac{|x-y|^{2}}{4 t}} \psi(y) d y .
$$

This immediately gives rise to the dispersive estimate

$$
\left\|e^{-i t \Delta} \psi\right\|_{\infty} \leq(4 \pi|t|)^{-\frac{1}{2}}\|\psi\|_{1} .
$$

Such an estimate cannot be true in general for the perturbed operator $H=$ $-\Delta+V(x)$. Even small perturbations of the Laplacian may lead to the formation of bound states, i.e. functions $f_{j} \in L^{2}$ satisfying $H f_{j}=-E_{j} f_{j}$. Bound states with strictly negative energy are known to possess exponential decay, hence they belong to the entire range of $L^{p}(\mathbb{R}), 1 \leq p \leq \infty$. For each of these bound states $f_{j}$, the associated evolution $e^{i t H} f_{j}=e^{-i t E_{j}} f_{j}$ clearly violates (1).

It is well known [3, 10, that if $V \in L^{1}(\mathbb{R})$, then the pure-point spectrum of $H$ consists of at most countably many eigenvalues $-E_{j}<0$. The absolutely continuous spectrum of $H$ is the entire positive half-line, and there is no singular continuous spectrum. Bound states can therefore be removed easily via a spectral projection, suggesting that one should look instead for dispersive estimates of the form

$$
\left\|e^{i t H} P_{a c}(H) \psi\right\|_{\infty} \lesssim|t|^{-\frac{1}{2}}\|\psi\|_{1} .
$$

The condition $V \in L^{1}$ does not always guarantee regularity at the endpoint of the continuous spectrum. We say that zero is a resonance of $H$ if there exists a bounded solution to the equation $H f=0$. Since resonances are not removed by the

Received by the editors June 12, 2006.

2000 Mathematics Subject Classification. Primary 35Q40; Secondary 34L25.

Key words and phrases. Schrödinger equation, dispersive estimates, transport, Jost solutions, scattering theory. 
spectral projection $P_{a c}(H)$, the validity of dispersive estimates invariably depends on whether zero is a resonance of $H$. Weder 12 and Goldberg-Schlag 5 have shown that (2) holds for all potentials with $(1+|x|)^{2} V \in L^{1}$, and that $(1+|x|) V \in L^{1}$ suffices provided zero is not a resonance.

The relatively slow time-decay of these estimates (the tail of the function $t^{-\frac{1}{2}}$ is not integrable) makes them unsuitable for many applications. We are therefore interested proving a dispersive estimate which improves the rate of decay by mapping between favorably weighted spaces. Statements of this type appear in the work of Murata [9] and Buslaev-Perelman [2, with weighted $L^{2}(\mathbb{R})$ as the underlying space. A weighted $L^{1} \rightarrow L^{\infty}$ bound was proven recently by Schlag [11. Our first theorem is a refinement of Schlag's result.

Theorem 1. Suppose $(1+|x|)^{3} V \in L^{1}(\mathbb{R})$ and zero is not a resonance of $H$. The continuous part of the Schrödinger evolution satisfies the bound

$$
\left\|(1+|x|)^{-1} e^{i t H} P_{a c}(H) \psi\right\|_{\infty} \lesssim|t|^{-\frac{3}{2}}\|(1+|x|) \psi\|_{1} .
$$

Recall that $\left|e^{i t H} P_{a c}(H) \psi\right|(x)$ is always dominated by $|t|^{-\frac{1}{2}}$, by (2). The additional estimate (3) reduces the bound even further for all $|x| \ll|t|$. This suggests that solutions experience transport away from the origin with nonzero velocity.

The assumption that zero energy is not a resonance is a necessary part of Theorem 1. To give an explicit example, consider the case $V=0$ with inital data $\psi(x)=e^{-\frac{|x|^{2}}{2}}$. For each $t$, the solution $e^{-i t \Delta} \psi(x)=(4 \pi(1-i t))^{-\frac{1}{2}} e^{\frac{-|x|^{2}}{2(1-i t)}}$ satisfies (2) but clearly violates (3). There is a significant degree of structure to a resonance at $\lambda=0$, as is seen in the power-series resolvent expansion of Jensen-Nenciu [7]:

$$
(H-(\lambda+i 0))^{-1}=\lambda^{-\frac{1}{2}} C_{-1}+C_{0}+\lambda^{\frac{1}{2}} C_{1}+\mathcal{O}(\lambda) .
$$

Here $C_{-1}$ is a projection onto the subspace spanned by the bounded solution of $H f=0$, or is vacuous if zero is not a resonance. One consequence is that the worst time-decay must be confined to a rank-one subspace of functions. More precisely, in the one-dimensionsal setting we prove the following:

Theorem 2. Suppose $(1+|x|)^{4} V \in L^{1}(\mathbb{R})$ and there is a nontrivial bounded function $f_{0}$ for which $H f_{0}=0$, normalized so that $\lim _{x \rightarrow \infty}\left(\left|f_{0}(x)\right|^{2}+\left|f_{0}(-x)\right|^{2}\right)=2$. Denote by $P_{0}$ the projection onto the span of $f_{0}$ given formally by $P_{0} \psi=\left\langle\psi, f_{0}\right\rangle f_{0}$.

The continuous part of the Schrödinger evolution satisfies the bound

$$
\left\|(1+|x|)^{-2}\left(e^{i t H} P_{a c}(H)-(-4 \pi i t)^{-\frac{1}{2}} P_{0}\right) \psi\right\|_{\infty} \lesssim|t|^{-\frac{3}{2}}\left\|\left(1+|x|^{2}\right) \psi\right\|_{1} .
$$

The proof of each theorem relies on a decomposition of the propagator $e^{i t H} P_{a c}(H)$ according the the spectral measure of $H$. Written this way,

$$
e^{i t H} \operatorname{Pac}(H) \psi=\int_{0}^{\infty} e^{i t \lambda} E_{a c}(d \lambda) \psi d \lambda
$$

where $E_{a c}(d \lambda)$ denotes the absolutely continuous part of the spectral measure of $H$. Since $V$ is assumed to be integrable, it is correct to assume that the absolutely continuous spectrum is supported on the interval $[0, \infty)$. The Stone formula provides additional information about the nature of $E_{a c}(d \lambda)$, namely

$$
\left\langle E_{a c}(d \lambda) f, g\right\rangle=\frac{1}{2 \pi i}\left\langle\left[R_{V}^{+}(\lambda)-R_{V}^{-}(\lambda)\right] f, g\right\rangle
$$


where $R_{V}^{ \pm}(\lambda):=(-\Delta+V-(\lambda \pm i 0))^{-1}$ is the continuation of the resolvent onto the positive real half-line. Substituting this into the previous equation yields

$$
\left\langle e^{i t H} P_{a c}(H) \psi, \varphi\right\rangle=\frac{1}{2 \pi i} \int_{0}^{\infty} e^{i t \lambda}\left\langle\left[R_{V}^{+}(\lambda)-R_{V}^{-}(\lambda)\right] \psi, \phi\right\rangle d \lambda
$$

It is convenient to make the change of variables $\lambda \mapsto \lambda^{2}$. For the purpose of changing variables inside the resolvent, recall that $R_{V}^{+}(\lambda)$ is an analytic continuation of the operator-valued function $(H-z)^{-1}$ from the upper half-plane. The continuation of $\left(H-z^{2}\right)^{-1}$ is therefore $\left(H-(\lambda+i 0)^{2}\right)^{-1}$, which is identical to $R_{V}^{+}\left(\lambda^{2}\right)$ along the positive half-line and $R_{V}^{-}\left(\lambda^{2}\right)$ along the negative half-line. This allows us to open up the domain of integration to the entire real line:

$$
\left\langle e^{i t H} P_{a c}(H) \psi, \varphi\right\rangle=\frac{1}{\pi i} \int_{-\infty}^{\infty} e^{i t \lambda^{2}} \lambda\left\langle R_{V}^{+}\left(\lambda^{2}\right) \psi, \varphi\right\rangle d \lambda
$$

For large values of $\lambda$ we will regard $R_{V}^{+}\left(\lambda^{2}\right)$ as a perturbation of the free resolvent $R_{0}^{+}\left(\lambda^{2}\right)$, which can be expressed explicitly as a convolution. This part of the argument has appeared previously in [11] and requires no further modification. For small $\lambda$ we will characterize the resolvent in terms of the Jost solutions of $H$. The desired estimates will follow from scattering-theory results of Deift and Trubowitz 4, using similar arguments to those in Goldberg-Schlag [5].

To separate the cases of low and high energy, let $\chi$ be a smooth even cutoff function that is equal to one when $|x| \leq \lambda_{0}$ and is supported on the interval where $|x| \leq 2 \lambda_{0}$. The value of $\lambda_{0}$ will be determined later, and depends primarily on the size of $V$. We will adopt the following notation for discussing polynomially weighted $L^{p}$ spaces:

$$
\begin{aligned}
\langle x\rangle & :=\left(1+|x|^{2}\right)^{\frac{1}{2}} \\
\|f\|_{L_{\sigma}^{p}} & :=\left\|\langle x\rangle^{\sigma} f\right\|_{L^{p}} .
\end{aligned}
$$

\section{High energy estimates}

Both Theorem 1 and Theorem 2 rely on the same estimate for the high-energy part of the evolution. This result can be found in 11, but we include it here for the sake of completeness.

Proposition 3. Assume that $V \in L_{1}^{1}(\mathbb{R})$ and choose $\lambda_{0} \geq\|V\|_{1}$. The following estimate is valid for all functions $\psi, \varphi \in L_{1}^{1}(\mathbb{R})$ :

$$
\left\langle e^{i t H}(1-\chi(\sqrt{H})) \psi, \varphi\right\rangle \lesssim|t|^{-\frac{3}{2}}\|\langle x\rangle \psi\|_{1}\|\langle x\rangle \varphi\|_{1} .
$$

Proof. By the same spectral argument that led to (5), here we are estimating the integral

$$
\frac{1}{\pi i} \int_{-\infty}^{\infty} e^{i t \lambda^{2}} \lambda(1-\chi(\lambda))\left\langle R_{V}^{+}\left(\lambda^{2}\right) \psi, \varphi\right\rangle d \lambda
$$

Integrate by parts once to obtain

$$
\frac{-1}{2 \pi t} \int_{-\infty}^{\infty} e^{i t \lambda^{2}} \frac{d}{d \lambda}\left[(1-\chi(\lambda))\left\langle R_{V}^{+}\left(\lambda^{2}\right) \psi, \varphi\right\rangle\right] d \lambda .
$$

The perturbed resolvent $R_{V}^{+}\left(\lambda^{2}\right)$ can be linked to the free resolvent $R_{0}^{+}\left(\lambda^{2}\right)$ via the identity $R_{V}^{+}\left(\lambda^{2}\right)=R_{0}^{+}\left(\lambda^{2}\right)\left(I+V R_{0}^{+}\left(\lambda^{2}\right)\right)^{-1}$, leading to the Born series 
expansion

$$
R_{V}^{+}\left(\lambda^{2}\right)=\sum_{k=0}^{\infty} R_{0}^{+}\left(\lambda^{2}\right)\left(V R_{0}^{+}\left(\lambda^{2}\right)\right)^{k} .
$$

The free resolvent $R_{0}^{+}\left(\lambda^{2}\right)$ has an explicit representation as an integral operator with kernel $K(x, y)=(2 i \lambda)^{-1} e^{i \lambda|x-y|}$. Substituting this into the identity above leads to the expression

$$
\begin{aligned}
\left\langle R_{V}^{+}\left(\lambda^{2}\right) \psi, \varphi\right\rangle=\sum_{k=0}^{\infty}(2 i \lambda)^{-(k+1)} & \int_{\mathbb{R}^{k+2}} e^{i \lambda\left(\sum_{j=0}^{k}\left|x_{j+1}-x_{j}\right|\right)} \\
& \psi\left(x_{0}\right) V\left(x_{1}\right) V\left(x_{2}\right) \ldots V\left(x_{k}\right) \varphi\left(x_{k+1}\right) d x_{0} \ldots d x_{k+1}
\end{aligned}
$$

which is a convergent series provided $2|\lambda| \geq\|V\|_{1}$. The absence of a boundary term in the integration by parts (7) is justified by a similar argument.

When this is substituted back into the integral (7) the differentiation in $\lambda$ leads to two distinct terms. Up to a constant factor, we have

$\left\langle e^{i t H}(1-\chi(\sqrt{H})) \psi, \varphi\right\rangle=$

$$
\begin{aligned}
\frac{1}{t} \int_{\mathbb{R}^{k+3}} \sum_{k=0}^{\infty} \sum_{m=0}^{k} e^{i t \lambda^{2}} e^{i \lambda\left(\sum_{j=0}^{k}\left|x_{j+1}-x_{j}\right|\right)} \frac{i(1-\chi(\lambda))}{(2 i \lambda)^{k+1}}\left|x_{m+1}-x_{m}\right| \\
\quad \times \psi\left(x_{0}\right) V\left(x_{1}\right) \ldots V\left(x_{k}\right) \varphi\left(x_{k+1}\right) d x_{0} \ldots d x_{k+1} d \lambda
\end{aligned}
$$

(86 a ) $-\frac{1}{t} \int_{\mathbb{R}^{k+3}} \sum_{k=0}^{\infty} e^{i t \lambda^{2}} e^{i \lambda\left(\sum_{j=0}^{k}\left|x_{j+1}-x_{j}\right|\right)}\left[\frac{(k+1)(1-\chi(\lambda))}{\lambda(2 i \lambda)^{k+1}}+\frac{\chi^{\prime}(\lambda)}{(2 i \lambda)^{k+1}}\right]$

$$
\times \psi\left(x_{0}\right) V\left(x_{1}\right) \ldots V\left(x_{k}\right) \varphi\left(x_{k+1}\right) d x_{0} \ldots d x_{k+1} d \lambda .
$$

In each of the terms we may rearrange the order of integration to handle the $d \lambda$ integral first (for the $k=0$ term in (8) this requires restricting to compact support in $\lambda$ and taking limits; otherwise it is permitted by Fubini's theorem). Evaluate this integral using Plancherel's identity: The Fourier transform of the oscillatory component $e^{i t \lambda^{2}} e^{i \lambda \sum\left|x_{j+1}-x_{j}\right|}$ is bounded above by $|t|^{-\frac{1}{2}}$ uniformly in the choice of all $x_{j}$. The Fourier transform of each expression involving the cutoff function (e.g. $\left.\frac{1-\chi\left(\lambda^{2}\right)}{(2 \lambda)^{k+1}}\right)$ is integrable with $L^{1}(\mathbb{R})$ norm bounded by $k\left(2 \lambda_{0}\right)^{-(k+1)}$. This implies that

$$
\begin{aligned}
\left|\left\langle e^{i t H}(1-\chi(\sqrt{H})) \psi, \varphi\right\rangle\right| \lesssim|t|^{-\frac{3}{2}} \sum_{k=0}^{\infty} k\left(2 \lambda_{0}\right)^{-(k+1)} & \\
& \times \int_{\mathbb{R}^{k+2}} \sum_{m=0}^{k}\left\langle x_{m+1}-x_{m}\right\rangle\left|\psi\left(x_{0}\right) V\left(x_{1}\right) \ldots V\left(x_{k}\right) \varphi\left(x_{k+1}\right)\right| d x_{0} \ldots d x_{k+1} .
\end{aligned}
$$

The sum of differences $\left\langle x_{m+1}-x_{m}\right\rangle$ can be controlled by $2 \sum_{m=0}^{k}\left\langle x_{m}\right\rangle$ using the triangle inequality. The inner integral is then separable, with the eventual bound

$$
\begin{aligned}
\left|\left\langle e^{i t H}(1-\chi(\sqrt{H})) \psi, \varphi\right\rangle\right| & \lesssim|t|^{-\frac{3}{2}} \sum_{k=0}^{\infty} k^{2}\left(2 \lambda_{0}\right)^{-k-1}\|V\|_{1}^{k-1}\|\langle x\rangle V\|_{1}\|\langle x\rangle \psi\|_{1}\|\langle x\rangle \varphi\|_{1} \\
& \lesssim \lambda_{0}^{-1}|t|^{-\frac{3}{2}}\|\langle x\rangle \psi\|_{1}\|\langle x\rangle \varphi\|_{1}
\end{aligned}
$$

provided $\lambda_{0} \geq\|V\|_{1}$. 


\section{LOW ENERGY ESTIMATES}

It remains to control the behavior of $e^{i t H} \chi(\sqrt{H}) \psi$, with the result depending on whether or not $H$ has a resonance at zero. The Born series used previously cannot be made to converge, so we rely instead on a characterization of the resolvent in terms of Jost solutions. For each $\lambda \in \mathbb{R}$, let $f_{ \pm}(x, \lambda)$ be the unique functions which satisfy

$$
-f_{ \pm}^{\prime \prime}(x, \lambda)+\left(V(x)-\lambda^{2}\right) f_{ \pm}(x, \lambda)=0, \quad f_{ \pm}(x, \lambda)=e^{ \pm i \lambda x} \text { as } x \rightarrow \pm \infty
$$

and $W(\lambda):=W\left[f_{+}(\cdot, \lambda), f_{-}(\cdot, \lambda)\right]$ be their Wronskian. Also define the Wronskian $\tilde{W}(\lambda)=W\left[f_{-}(\cdot, \lambda), f_{+}(\cdot,-\lambda)\right]$. The perturbed resolvent $R_{V}\left(\lambda^{2}\right)$ is an integral operator whose kernel is given by

$$
R_{V}^{+}\left(\lambda^{2}\right)(x, y)=\frac{f_{+}(x, \lambda) f_{-}(y, \lambda)}{W(\lambda)}
$$

for all $x \geq y$, and is symmetric for $x<y$.

Note that $f_{ \pm}(\cdot,-\lambda)$ solve the same second-order differential equation as $f_{ \pm}(\cdot, \lambda)$, hence they must be linearly dependent. The coefficients in the relation

$$
f_{-}(x, \lambda)=\alpha(\lambda) f_{+}(x, \lambda)+\beta(\lambda) f_{+}(x,-\lambda)
$$

are given by $\alpha(\lambda)=\frac{\tilde{W}(\lambda)}{-2 i \lambda}$ and $\beta(\lambda)=\frac{W(\lambda)}{-2 i \lambda}$. These in turn are closely linked to the reflection and transmission coefficients, namely: $\alpha(\lambda)=\frac{R_{1}(\lambda)}{T(\lambda)}$ and $\beta(\lambda)=\frac{1}{T(\lambda)}$. Conjugate symmetry requires that $\beta(-\lambda)=\overline{\beta(\lambda)}$ and $\alpha(-\lambda)=\overline{\alpha(\lambda)}$. Conservation of energy additionally requires that $|\alpha(\lambda)|^{2}+1=|\beta(\lambda)|^{2}$ for every value of $\lambda$.

Since $\beta(\lambda)$ is always positive, $W(\lambda)$ cannot vanish except possibly when $\lambda=0$. The condition $W(0)=0$ is satisfied precisely if zero is a resonance; in the generic (nonresonant) case the values of $W(\lambda)$ are everywhere nonzero.

It is common to rewrite the Jost solutions as $f_{ \pm}(x, \lambda)=e^{ \pm i \lambda x} m_{ \pm}(x, \lambda)$, where $m_{ \pm}(x, \lambda) \rightarrow 1$ as $x \rightarrow \pm \infty$. The relevant properties of the functions $m_{ \pm}(x, \lambda)$ are summarized below. See [4, Lemma 3 for details.

Lemma 4. Suppose $V \in L_{\sigma}^{1}, \sigma \geq 1$. For each $x$ the functions $m_{ \pm}(x, \cdot)-1$ belong to the Hardy space $H^{2+}$ of analytic functions on the upper half-plane. Consequently, their Fourier transform in the second variable, denoted by $m_{ \pm}(x, \hat{\rho})$, is supported on the halfine $\rho \geq 0$.

Define $I(\rho):=\int_{|t|>\rho}|V(t)| d t$. The following pointwise estimates for $m_{ \pm}(x, \hat{\rho})$ are valid over the specified ranges of $x$ and all $\rho>0$ :

$$
\begin{aligned}
\text { If } x \geq 0 \text {, then }\left\{\begin{array}{l}
\left|m_{+}(x, \hat{\rho})-\delta_{0}(\rho)\right| \lesssim I(\rho), \\
\left|\frac{\partial}{\partial x} m_{+}(x, \hat{\rho})\right| \lesssim I(\rho)+|V(x+\rho)|, \\
\left|\frac{\partial}{\partial \rho} m_{+}(x, \hat{\rho})\right| \lesssim I(\rho)+|V(x+\rho)| .
\end{array}\right. \\
\text { If } x \leq 0 \text {, then }\left\{\begin{array}{l}
\left|m_{-}(x, \hat{\rho})-\delta_{0}(\rho)\right| \lesssim I(\rho), \\
\left|\frac{\partial}{\partial x} m_{-}(x, \hat{\rho})\right| \lesssim I(\rho)+|V(x-\rho)|, \\
\left|\frac{\partial}{\partial \rho} m_{-}(x, \hat{\rho})\right| \lesssim I(\rho)+|V(x-\rho)| .
\end{array}\right.
\end{aligned}
$$

It follows that each of the above functions involving $m_{ \pm}(x, \hat{\rho})$ belongs to $L_{\sigma-1}^{1}(\mathbb{R})$, uniformly over all $x$ in the appropriate halfline. Furthermore, the Fourier transform of $\partial_{\lambda} m_{ \pm}(x, \cdot)$ belongs to $L_{\sigma-2}^{1}(\mathbb{R})$. 
Corollary 5. Suppose $V \in L_{\sigma}^{1}(\mathbb{R}), \sigma \geq 1$ and let $\tilde{\chi}(\lambda)=\chi\left(\frac{\lambda}{4}\right)$. The functions $\tilde{\chi}(\lambda) W(\lambda)$ and $\tilde{W}(\lambda)$ both have Fourier transform (with respect to $\lambda$ ) in the space $L_{\sigma-1}^{1}(\mathbb{R})$.

Proof. Recall that $f_{ \pm}(x, \lambda)=e^{ \pm i \lambda x} m_{ \pm}(x, \lambda)$. By this definition,

$$
\begin{array}{r}
\tilde{\chi}(\lambda) W(\lambda)=\tilde{\chi}(\lambda)\left(m_{+}(0, \lambda) \partial_{x} m_{-}(0, \lambda)-\partial_{x} m_{+}(0, \lambda) m_{-}(0, \lambda)\right) \\
-2 i \lambda \tilde{\chi}(\lambda) m_{+}(0, \lambda) m_{-}(0, \lambda)
\end{array}
$$

and

$$
\tilde{W}(\lambda)=m_{-}(0, \lambda) \partial_{x} m_{+}(0,-\lambda)-\partial_{x} m_{-}(0, \lambda) m_{+}(0,-\lambda) .
$$

According to the pointwise bounds in (11), each individual function $m_{ \pm}(0, \pm \lambda)$ has Fourier transform in $L_{\sigma-1}^{1}$, which is an algebra with respect to convolutions.

Proof of Theorem 1. The desired bounds have already been established in the high energy case by Proposition 3. The remaining task is to evaluate the part of the integral not considered in (7), namely

$$
\frac{-1}{2 \pi t} \int_{-\infty}^{\infty} e^{i t \lambda^{2}} \frac{d}{d \lambda}\left[\chi(\lambda)\left\langle R_{V}^{+}\left(\lambda^{2}\right) \psi, \varphi\right\rangle\right] d \lambda .
$$

After applying the formula (9) for the integral kernel of $R_{V}^{+}\left(\lambda^{2}\right)$ and Plancherel's identity, it suffices to show that the Fourier transform (in $\lambda$ ) of

$$
\frac{d}{d \lambda}\left[\chi(\lambda) \frac{f_{-}(x, \lambda) f_{+}(y, \lambda)}{\tilde{\chi}(\lambda) W(\lambda)}\right]
$$

belongs to $L^{1}(\mathbb{R})$ with norm bounded by $\langle x\rangle\langle y\rangle$ for all choices of $x \leq y$. The correct estimate will also hold for $x>y$ by symmetry of the resolvent.

First consider the case $x \leq 0 \leq y$. We are interested in the Fourier transform of the function

$$
\begin{aligned}
i(y-x) \frac{e^{i(y-x)(\cdot)} \chi m_{-}(x, \cdot) m_{+}(y, \cdot)}{\tilde{\chi} W} & +\frac{e^{i(y-x)(\cdot)} \partial_{\lambda}\left[\chi m_{-}(x, \cdot) m_{+}(y, \cdot)\right]}{\tilde{\chi} W} \\
& -\frac{e^{i(y-x)(\cdot)} \chi m_{-}(x, \cdot) m_{+}(y, \cdot) \partial_{\lambda}[\tilde{\chi} W]}{(\tilde{\chi} W)^{2}} .
\end{aligned}
$$

Lemma 4 ensures that the Fourier transform of each numerator has $L^{1}$ norm bounded uniformly in $x \leq 0 \leq y$. If zero is not a resonance, then the Wronskian $W(\lambda)$ is everywhere nonzero. The Wiener Lemma (see, for example, [8], Chapter VIII) then implies that $\chi\left(\frac{\lambda}{2}\right)(\tilde{\chi} W)^{-1}$ also has integrable Fourier transform, making the division possible as well. Collectively, the $L^{1}$ norm of the Fourier transform will be bounded by $|y-x|$ plus a constant, which in turn is bounded by $\langle x\rangle\langle y\rangle$.

In the case $0<x<y$, there is no uniform control over quantities derived from $m_{-}(x, \lambda)$. To avoid this problem, use the intertwining coefficients to write

$$
\begin{aligned}
f_{-}(x, \lambda)= & \alpha(\lambda) f_{+}(x, \lambda)+\beta(\lambda) f_{-}(x,-\lambda) \\
= & \frac{-1}{4 i}\left[\frac{\tilde{W}(\lambda)+W(\lambda)}{\lambda}\left(f_{+}(x, \lambda)+f_{+}(x,-\lambda)\right)\right. \\
& \left.+(\tilde{W}(\lambda)-W(\lambda)) e^{i \lambda x} \frac{m_{+}(x, \lambda)-m_{+}(x,-\lambda)}{\lambda}\right] .
\end{aligned}
$$


The only functions here of any concern are the expressions with $\lambda$ in the denominator. Observe that not only is $m_{+}(x, \hat{\rho})-m_{+}(-\hat{\rho}) \in L_{\sigma-1}^{1}(\mathbb{R})$, but its integral over the real line is exactly zero. Because of this, the Fourier transform of $\frac{m_{+}(x, \lambda)-m_{+}(x,-\lambda)}{\lambda}$ is given by

$$
\left[\frac{m_{+}(x, \cdot)-m_{+}(x,-(\cdot))}{(\cdot)}\right]^{\wedge}(s)=-i \int_{s}^{\infty} m_{+}(x, \hat{\rho})-m_{+}(x,-\hat{\rho}) d \rho
$$

which belongs to $L_{\sigma-2}^{1}(\mathbb{R})$ uniformly in $x \geq 0$. An identical argument holds for the fraction $\frac{\tilde{W}(\lambda)+W(\lambda)}{\lambda}$ by expanding out each Wronskian according to its definition. One can recognize this as a restatement of the well-known fact about reflection coefficients at zero energy: $R_{1}(0)=-1$.

To complete the calculations as in the previous case, one may need to deal with derivatives such as $\frac{d}{d \lambda}\left[\frac{\tilde{W}(\lambda)+W(\lambda)}{\lambda}\right]$. The Fourier transform of such a function is in $L_{\sigma-3}^{1}(\mathbb{R})$, which is still integrable provided $\sigma \geq 3$. Depending on where else the derivative in (12) may fall, one obtains norm bounds of size $\langle x\rangle+\langle y\rangle+1$, which is again bounded by $\langle x\rangle\langle y\rangle$.

The case $x \leq y<0$ is handled in an identical way by using the intertwining relation $f_{+}(y, \lambda)=-\overline{\alpha(\lambda)} f_{-}(y, \lambda)+\beta(\lambda) f_{-}(y,-\lambda)$ instead of (10).

Proof of Theorem 2. All of the estimates in Lemma 4 are still valid in the resonant case. The one fundamental difference is that $W(\lambda)$ vanishes when $\lambda=0$ (and at no other $\lambda \in \mathbb{R}$ ). Consequently, the functions $\alpha(\lambda)$ and $\beta(\lambda)=\frac{W(\lambda)}{-2 i \lambda}$ are both continuous and real-valued at the origin. The Fourier transforms of $\alpha$ and $\tilde{\chi} \beta$ lie in the space $L_{\sigma-2}^{1}(\mathbb{R})$, and moreover $\beta(\lambda) \neq 0$ over the entire real line.

Thanks to the resonance, the integral

$$
\frac{1}{2 \pi} \int_{-\infty}^{\infty} e^{i t \lambda^{2}} \chi(\lambda) \frac{f_{-}(x, \lambda) f_{+}(y, \lambda)}{\tilde{\chi}(\lambda) \beta(\lambda)} d \lambda
$$

must have a stationary phase contribution on the order of $|t|^{-1 / 2}$. The integrand is sufficently regular such that one can isolate the leading term

$$
\begin{aligned}
\frac{1}{2 \pi} \int_{-\infty}^{\infty} e^{i t \lambda^{2}} \frac{f_{-}(x, 0) f_{+}(y, 0)}{\beta(0)} d \lambda & =(-4 \pi i t)^{-\frac{1}{2}} \frac{f_{-}(x, 0) f_{+}(y, 0)}{\beta(0)} \\
& =(-4 \pi i t)^{-\frac{1}{2}} f_{0}(x) f_{0}(y)
\end{aligned}
$$

leaving a remainder of order $|t|^{-\frac{3}{2}}$. It is clear that $f_{-}(\cdot, 0)$ and $f_{+}(\cdot, 0)$ are both scalar multiples of $f_{0}$. The limiting values of $f_{-}(x, 0)$ as $x \rightarrow \pm \infty$ are $\beta(0)+\alpha(0)$ and 1 , respectively. This makes

$$
f_{-}(x, 0)=\sqrt{1+(\beta(0)+\alpha(0))^{2}} f_{0}(x) .
$$

A similar argument shows that $f_{+}(y, 0)=\sqrt{1+(\beta(0)-\alpha(0))^{2}} f_{0}(y)$. The two square roots have signs in common if $\beta(0)>0$ and are of opposite sign if $\beta(0)<0$. The last line of (14) is obtained from this fact and the identity $\alpha^{2}(0)+1=\beta^{2}(0)$.

The remainder term is given explicitly by

$$
\frac{1}{2 \pi} \int_{-\infty}^{\infty} e^{i t \lambda^{2}}\left(G_{x, y}(\lambda)-G_{x, y}(0)\right) d \lambda=\frac{1}{4 \pi i t} \int_{-\infty}^{\infty} e^{i t \lambda^{2}} \frac{d}{d \lambda}\left[\frac{G_{x, y}(\lambda)-G_{x, y}(0)}{\lambda}\right] d \lambda
$$


where

$$
\begin{aligned}
G_{x, y}(\lambda) & =e^{i \lambda(y-x)} \chi(\lambda) \frac{m_{-}(x, \lambda) m_{+}(y, \lambda)}{\tilde{\chi}(\lambda) \beta(\lambda)} \\
& =e^{i \lambda(y+x)} \frac{\chi(\lambda) \alpha(\lambda) m_{+}(x, \lambda) m_{+}(y, \lambda)}{\tilde{\chi}(\lambda) \beta(\lambda)}+e^{i \lambda(y-x)} \chi(\lambda) m_{+}(x,-\lambda) m_{+}(y, \lambda) .
\end{aligned}
$$

One uses the first formula for $G_{x, y}(\lambda)$ in the case $x \leq 0 \leq y$ and the second formula when $0<x \leq y$. There is a third formula, quite similar to the second, which is useful when $x \leq y<0$.

In order to complete the proof it suffices to bound the $L^{1}$ norm of the Fourier transform of $\frac{d}{d \lambda}\left[\frac{G_{x, y}(\lambda)-G_{x, y}(0)}{\lambda}\right]$ by the quantity $\langle x\rangle^{2}\langle y\rangle^{2}$. If we are using the second formula for $G_{x, y}$, it is permissible to bound each term separately. All of these estimates are consequences of the general rule stated below.

Proposition 6. Suppose the Fourier transform of $F(\lambda)$ belongs to $L_{2}^{1}(\mathbb{R})$. Define

$$
G(\lambda)=\frac{d}{d \lambda}\left[\frac{e^{i k \lambda} F(\lambda)-F(0)}{\lambda}\right] .
$$

The Fourier transform of $G$ is integrable, with the bound $\|\widehat{G}\|_{1} \lesssim\langle k\rangle^{2}\|\widehat{F}\|_{L_{2}^{1}}$.

$$
\text { Write out } G(\lambda)=\frac{d}{d \lambda}\left[\frac{e^{i k \lambda}-1}{\lambda}\right] F(\lambda)+\frac{e^{i k \lambda}-1}{\lambda} F^{\prime}(\lambda)+\frac{d}{d \lambda}\left[\frac{F(\lambda)-F(0)}{\lambda}\right] .
$$

The Fourier transforms of $\frac{e^{i k \lambda}-1}{\lambda}$ and its derivative are integrable, with norms proportional to $k$ and $k^{2}$ respectively. The Fourier transform of $\frac{F(\lambda)-F(0)}{\lambda}$ belongs to $L_{1}^{1}(\mathbb{R})$ (compare to (13) to see that this is controlled by $\|\widehat{F}\|_{L_{2}^{1}}$ ), and that of its derivative is integrable. By convolution in $L^{1}$, each of the three terms above will yield a bound no greater than $\langle k\rangle^{2}\|\widehat{F}\|_{L_{2}^{1}}$, as desired.

\section{REFERENCES}

1. Artbazar, G., Yajima, K. The $L^{p}$-continuity of wave operators for one dimensional Schrödinger operators. J. Math. Sci. Univ. Tokyo 7 (2000), no. 2, 221-240. MR 1768465 (2001f:34166)

2. Buslaev, V.S., Perelman, G. S. Scattering for the nonlinear Schrödinger equation: states that are close to a soliton, (Russian) Algebra i Analiz 4 (1992), no. 6, 63-102; translation in St. Petersburg Math. J. 4 (1993), no. 6, 1111-1142. MR1199635 (94b:35256)

3. Coddington, E. A., Levinson, N. Theory of ordinary differential equations, McGraw-Hill, New York, 1955. MR0069338(16:1022b)

4. Deift, P., Trubowitz, E. Inverse scattering on the line. Comm. Pure Appl. Math. XXXII (1979), 121-251. MR0512420 (80e:34011)

5. Goldberg, M., Schlag, W. Dispersive estimates for the Schrödinger operator in dimensions one and three, Comm. Math. Phys. 251 (2004), no. 3, 157-178. MR2096737 (2005g:81339)

6. Jensen, A., Kato, T. Spectral properties of Schrödinger operators and time-decay of the wave functions. Duke Math. J. 46 (1979), no. 3, 583-611. MR0544248 (81b:35079)

7. Jensen, A., Nenciu, G. A unified approach to resolvent expansions at thresholds. Rev. Math. Phys. 13 (2001), no. 6, 717-754. MR.1841744 (2002e:81031)

8. Katznelson, Y. An introduction to harmonic analysis. Dover, 1968. MR0422992 (54:10976)

9. Murata, M. Asymptotic expansions in time for solutions of Schrödinger-type equations, J. Funct. Anal. 49 (1) (1982), 10-56. MR0680855 (85d:35019)

10. Reed, M., Simon, B. Methods of modern mathematical physics. IV. Analysis of operators. Academic Press [Harcourt Brace Jovanovich, Publishers], New York-London, 1978. MR0493421 $(58: 12429 \mathrm{c})$

11. Schlag, W. Dispersive estimates for Schroedinger operators: a survey preprint 2004, to appear in Proceedings of the conference "Workshop in Aspects of Nonlinear PDEs," IAS Princeton. 
12. Weder, R. $L^{p}-L^{\dot{p}}$ estimates for the Schrödinger equation on the line and inverse scattering for the nonlinear Schrödinger equation with a potential. J. Funct. Anal. 170 (2000), no. 1, 37-68. MR1736195 (2001e:35135)

13. Weder, R. The $W_{k, p}$-continuity of the Schrödinger wave operators on the line. Comm. Math. Phys. 208 (1999), no. 2, 507-520. MR.1729096 (2001c:34178)

Department of Mathematics, Johns Hopkins University, 3400 N. Charles St., BaltiMORE, MaRYLAND 21218

E-mail address: mikeg@math.jhu.edu 